\title{
Supercontinuum generation and carrier envelope offset frequency measurement in a tapered single-mode fiber
}

\author{
Long Zhang • Hainian Han • Yanying Zhao • \\ Lei Hou $\cdot$ Zijiao Yu $\cdot$ Zhiyi Wei
}

Received: 12 June 2014/Accepted: 1 October 2014/Published online: 17 October 2014

(C) The Author(s) 2014. This article is published with open access at Springerlink.com

\begin{abstract}
We report supercontinuum generation by launching femtosecond $\mathrm{Yb}$-fiber laser pulses into a tapered single-mode fiber of $3-\mu \mathrm{m}$ core diameter. A spectrum of more than one octave, from 550 to $1,400 \mathrm{~nm}$, has been obtained with an output power of $1.3 \mathrm{~W}$ at a repetition rate of $250 \mathrm{MHz}$, corresponding to a coupling efficiency of up to $60 \%$. Using a typical $f$ - $2 f$ interferometer, the carrier envelope offset frequency was measured and found to have a signal-to-noise ratio of nearly $30 \mathrm{~dB}$.
\end{abstract}

\section{Introduction}

Spectral broadening based on nonlinear effects has received widespread attention due to its rich spectral content, excellent coherence and high spatial brightness. It has a broad range of applications in areas such as optical fiber communication [1], optical coherence tomography [2] and frequency metrology [3]. Of particular importance is its use in the measurement of the carrier envelope offset frequency $\left(f_{\text {ceo }}\right)$ of femtosecond optical frequency combs, wherein a spectrum extending over one optical octave is necessary when employing the $f$-2f interferometer technique.

L. Zhang $\cdot$ H. Han $(\bowtie) \cdot$ L. Hou $\cdot$ Z. Yu $\cdot$ Z. Wei

Beijing National Laboratory for Condensed Matter Physics, Institute of Physics, Chinese Academy of Sciences,

Beijing 100190, China

e-mail: hnhan@iphy.ac.cn

Z. Wei

e-mail: zywei@iphy.ac.cn

Y. Zhao

State Key Laboratory of Nuclear Physics and Technology, Peking University, Beijing 100871, China
It is usual to use a photonic crystal fiber (PCF) [4-9] to broaden the laser spectrum. Because of its special structure, it is easy to confine light in the small PCF core area and customize the dispersion characteristics. Broadband spectra of more than one octave have been generated with ultrashort laser pulses in PCF. An alternative scheme for spectrum broadening is fiber taper technology, which was first applied in conventional single-mode fiber and has since remained quite popular [10-14]. Compared with PCF, the tapered single-mode fiber has a natural double-sided funnel structure which enables light to be focused much more easily and thus generate a supercontinuum (SC) with higher efficiency and stability. Tapered single-mode fibers may therefore have great potential in developing state-of-the-art optical frequency combs, which demand stable, high power SC generation for reliable performance. On the other hand, the single-mode tapered fiber technique is quite simple, inexpensive and flexible for various applications. In 2000, Birks et al. reported spectrum broadening in a tapered singlemode fiber with a $2-\mu \mathrm{m}$ diameter in the central portion of a standard telecommunications fiber. As a result, an SC extending across a two-octave spectrum was achieved by using femtosecond laser pulses generated by Ti:sapphire [11] and Cr:forsterite [12] oscillators. In addition, the tapered single-mode fiber is able to support high power transmission up to several watts. For example, a multi-watt SC source was set up in cascaded tapered single-mode fibers of different core diameters [13]. An average output power of more than $5 \mathrm{~W}$ was obtained. When the core diameter is reduced to $<1.5 \mu \mathrm{m}$, the zero-dispersion wavelength (ZDW) matches well with visible light, which is beneficial for the generation of white-light SC [14]. Although many important applications, such as mode propagation, fourwave mixing and optical sensing have been realized by using tapered single-mode fibers [15-18]; to our 
knowledge, no measurements of the $f_{\text {ceo }}$ in an optical frequency comb have been reported before.

In this paper, we describe the generation of a $\mathrm{SC}$ and the measurement of $f_{\text {ceo }}$ by injecting high repetition rate femtosecond pulses into a tapered single-mode fiber. The laser pulses, generated by an ytterbium fiber (Yb-fiber) oscillator, were amplified and compressed to nearly 115 fs. An output power up to $2.2 \mathrm{~W}$ was achieved, corresponding to a single pulse energy of $8.8 \mathrm{~nJ}$ at a repetition rate of $250 \mathrm{MHz}$. Then the compressed pulses were coupled into a home-made tapered single-mode fiber with a core diameter of $3 \mu \mathrm{m}$ and taper length of $9 \mathrm{~cm}$. Because of the strong nonlinear effects in the tapered fiber, a spectrum covering over an octave, from 550 to $1,400 \mathrm{~nm}$, was obtained. The SC power was more than $1.3 \mathrm{~W}$ with a coupling efficiency of up to $60 \%$. Based on this SC spectrum, the $f_{\text {ceo }}$ was measured with a typical $f$-2f interferometer, which indicates that the tapered single-mode fiber could be a suitable choice for constructing optical frequency combs.

\section{Experiment}

\subsection{Properties of the tapered single-mode fiber}

The tapered fiber we used was made of a conventional single-mode fiber (SMF-28, Corning). After synchronous heating and pulling in a taper-drawing rig, a tapered section in the center of untreated single-mode fiber was fabricated. In our experiment, the fiber cladding was tapered from 125 to $3 \mu \mathrm{m}$ over a length of about $9 \mathrm{~cm}$. The transition length on either side of the tapered section was nearly $3.5 \mathrm{~cm}$, so the total length of the tapered single-mode fiber was $16 \mathrm{~cm}$. When light travels in the tapered section, it propagates within the whole cladding, rather than being confined to the core only. Because of the large index difference between the cladding and air, the light is guided quite well in the tapered fiber. In order to avoid touching other objects, the fiber was hung and enclosed in a plastic housing, to ensure low-loss transmission and easy handling.

According to previous analyses [19-21], the nonlinear coefficient largely depends on the fiber parameters, such as the core diameter, core-cladding index difference, and the effective core area of the transmission mode. When we only consider the fundamental mode transmission, the nonlinear coefficient is calculated to be about $53 \mathrm{~W}^{-1} \mathrm{~km}^{-1}$ at a wavelength of $1,030 \mathrm{~nm}$.

The dispersion characteristics of the tapered fiber were also estimated. The change of ZDW with core diameter is given in Fig. 1a, while the group velocity dispersion (GVD) and third order dispersion in the 3- $\mu \mathrm{m}$-core-diameter tapered fiber are shown in Fig. 1b. The GVD was about $-46 \mathrm{ps}^{2} / \mathrm{km}$ at $1,030 \mathrm{~nm}$. In this case, the third order dispersion is very small and has little impact on the nonlinear interaction between the femtosecond pulses and fiber, so it may be neglected.

Although it is obvious that a smaller core can enhance the nonlinearity and shift the ZDW to shorter wavelengths, it also restricts the spectrum range because of the large difference between the incident and ZDWs. In addition, when the core diameter is too small $(<2 \mu \mathrm{m})$, the fiber cannot sustain high power pulses, so we chose a $3-\mu \mathrm{m}$ tapered fiber to generate SC in our experiment.

\subsection{Experimental setup}

The experimental setup is shown in Fig. 2. It consists of a $250 \mathrm{MHz}$ femtosecond $\mathrm{Yb}$-fiber oscillator (MenloSystems $\mathrm{GmbH}$ ) and operates in the nonlinear polarization rotation
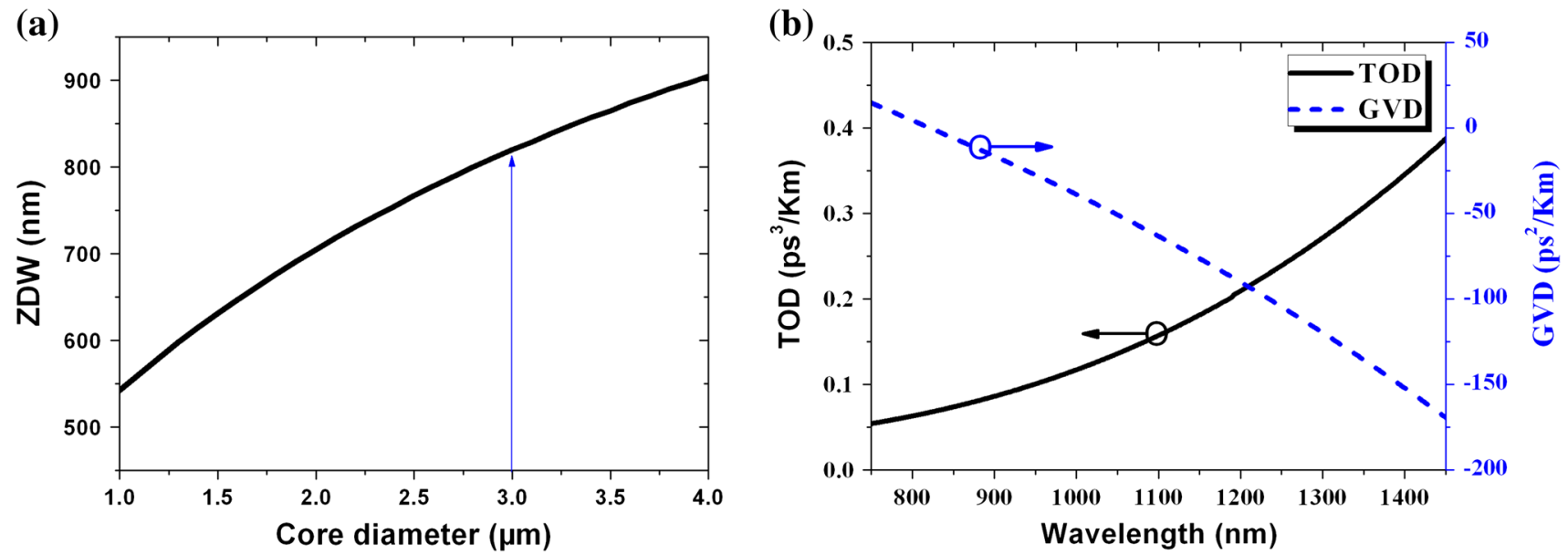

Fig. 1 a Zero-dispersion wave as a function of core diameter in the tapered single-mode fiber. b Calculated group velocity dispersion (GVD, blue, dashed curve) and third order dispersion (TOD, black, solid curve) versus wavelength for a core diameter of $3 \mu \mathrm{m}$ 
Fig. 2 Experimental setup of $\mathrm{SC}$ and $f_{\text {ceo }}$ generation with a tapered single-mode fiber. AS: aspherical lens, Yb:SCF/DCF: $\mathrm{Yb}$ doped single-clad/doubleclad fiber, HWP: half-wave plate, TGs: transmission gratings, BHR: broadband half reflective mirror (coating: 900-1,200 nm), DM: dichroic mirror, DDL: dispersion delay line, PBS: polarization beam splitter, RG: reflection grating, APD: avalanche photodetector

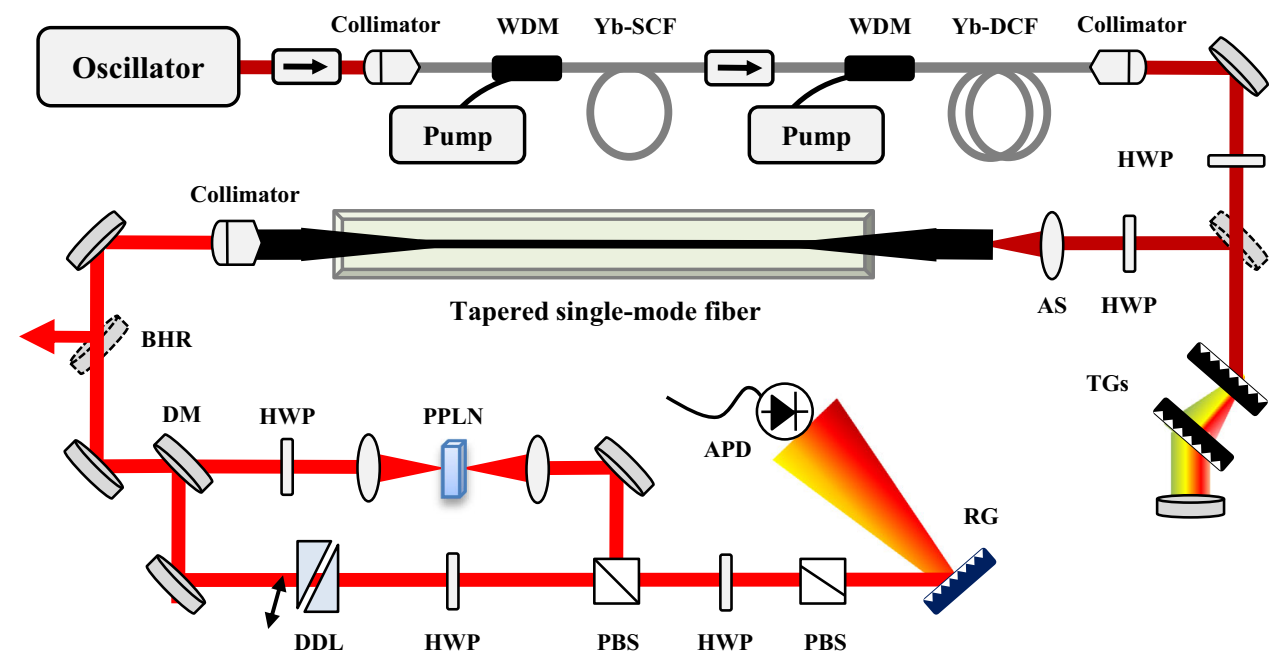

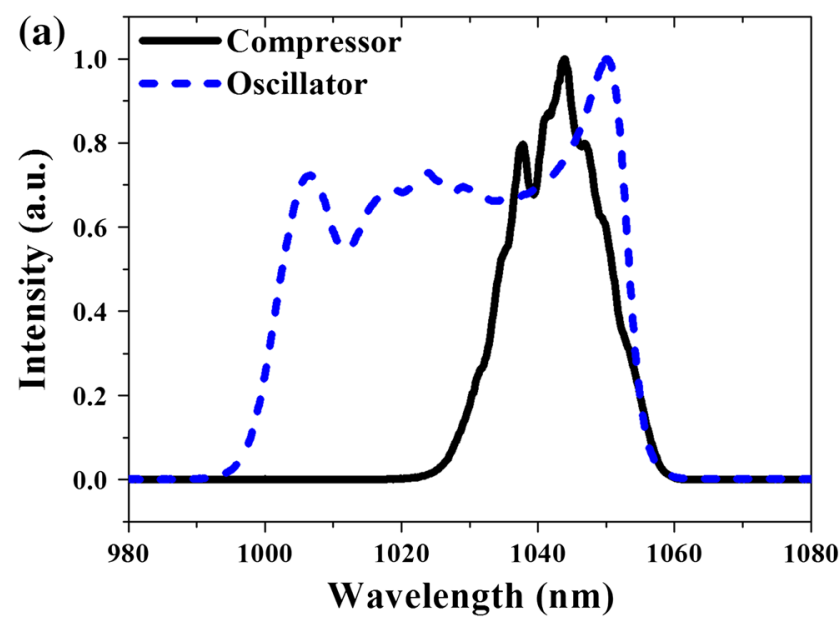

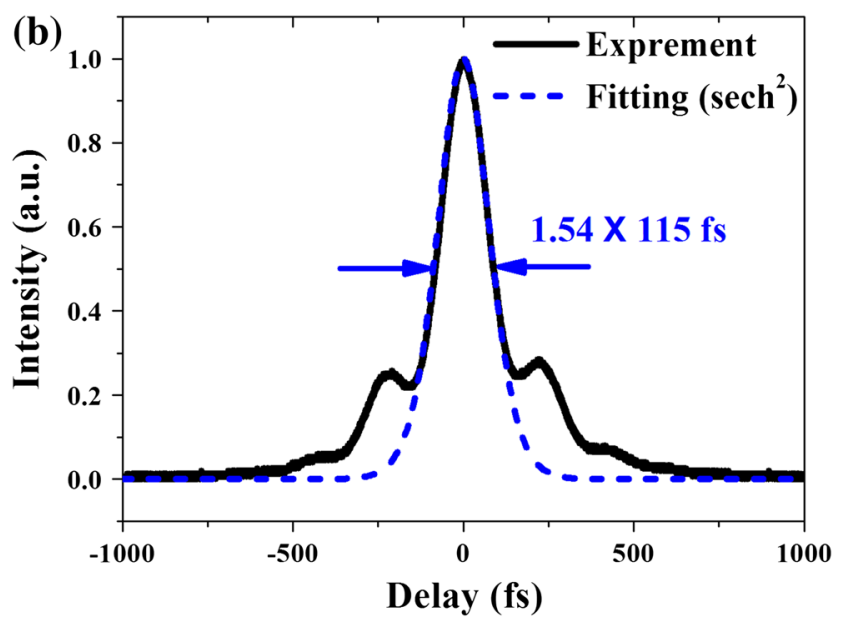

Fig. 3 a Normalized spectral intensity of the oscillator (blue dashed curve) and compressor (black solid curve). b Intensity autocorrelation curve after compressing (black solid curve) and $\operatorname{sech}^{2}$-fitting result (blue dashed curve)

mode-locked configuration. The spectral width is about $50 \mathrm{~nm}$ at the center wavelength of $1,030 \mathrm{~nm}$, as shown by the blue dashed curve in Fig. 3a. The laser output, emitted from an intra-cavity polarization beam splitter, is coupled into a fiber splitter and divided into three routes. One beam has nearly $30 \mathrm{~mW}$ average power and is led into a doublestage fiber amplifier. Optical isolators are inserted between the oscillator and amplifiers to avoid unnecessary damage caused by detrimental reflected light. After amplification, the pulses are compressed using a pair of transmission gratings. Due to the influence of gain narrowing during amplification, the intensity around the emission peak will be amplified effectively, so the spectral width after compressing is narrowed to about $15 \mathrm{~nm}$, as shown by the black solid curve in Fig. 3a. The intensity autocorrelation curve (Fig. $3 b$ ) shows that the pulse duration is about 115 fs for an amplified power of $2.2 \mathrm{~W}$.

\section{Results and discussion}

\subsection{Supercontinuum generation}

In order to obtain an octave-spanning spectrum, we used the 3- $\mu \mathrm{m}$-core-diameter, 9-cm long tapered single-mode fiber described above. The enclosed fiber was mounted on a three-dimension precision stage. The compressed pulses were focused into the fiber with an aspherical lens of 6.24$\mathrm{mm}$ focal length, and the output was collimated with an aspherical lens attached to a FC/APC patch cable. The output was measured with an optical power meter and spectrum analyzer (AQ 6315A, ANDO). The SC output as a function of input power is shown in Fig. 4a, where we see that the maximum transmitted power is $1.3 \mathrm{~W}$ for an input power of $2.2 \mathrm{~W}$. The coupling efficiency is about $60 \%$, and stable operation can be maintained for a long time by 

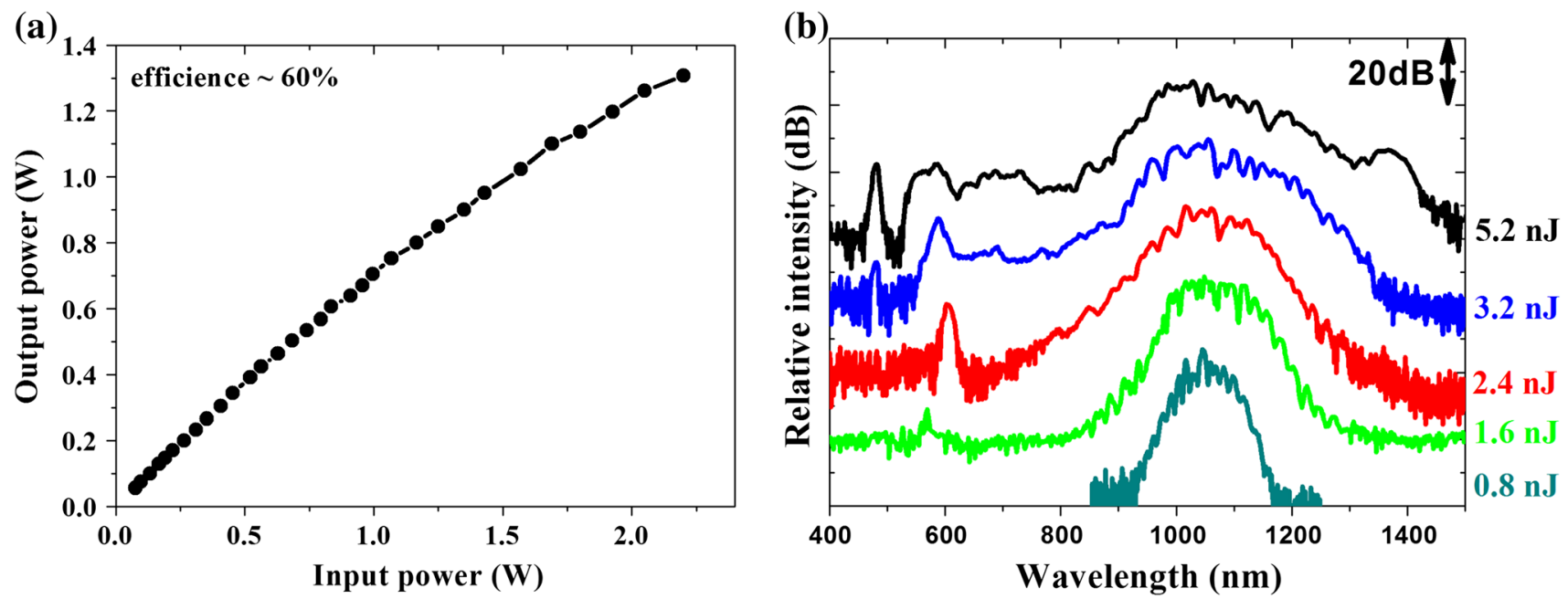

Fig. 4 a Supercontinuum output as a function of input power; the coupling efficiency is about $60 \%$. b Supercontinuum spectrum for different coupled pulse energies from 0.8 to $5.2 \mathrm{~nJ}$

reason of the large core diameter at the fiber end surfaces which do not have a honeycomb structure.

Figure $4 \mathrm{~b}$ shows the change in the SC spectrum for different input pulse energies, the values of which are indicated on the right vertical axis. It is evident that the spectral bandwidth broadens as the coupled energy increases. Nonlinear effects, such as soliton fission, Raman scattering and dispersion effects, play important roles during the evolution process [6]. When the pulse energy is $<0.8 \mathrm{~nJ}$, soliton fission plays a dominant role in the abnormal dispersion regime. As $\mathrm{s}$ result, symmetric broadening occurs and, accordingly, high order solitons are generated around $1,030 \mathrm{~nm}$. As the pulse energy increases, Raman perturbation will affect soliton production which will shift toward longer wavelengths, extending the spectrum from 1,100 to $1,400 \mathrm{~nm}$ [22]. On the other hand, visible components will gradually appear under the impact of dispersive wave radiation [23, 24]. The discrete peak around $600 \mathrm{~nm}$ is first observed at a coupled energy of 1.6 $\mathrm{nJ}$. When higher pulse energies are injected, more spectral components are induced between 550 to $900 \mathrm{~nm}$. The peak around $480 \mathrm{~nm}$ might be the second harmonic generated from the residual pump laser beam. Finally, at the maximum pulse energy of $5.2 \mathrm{~nJ}$, the SC span exceeds an octave, extending from 550 to $1,400 \mathrm{~nm}$. However, the shape of the SC spectrum is rather ragged across its range. The reason for this is probably that the ZDW of the tapered fiber we used is slightly offset from the incident wavelength, which results in more pulse energy being distributed to the near infrared region. In spite of this, the broadened spectrum can span more than one octave range and completely satisfy the needs for the high and low frequency components of $f_{\text {ceo }}$ detection in an $f$ $2 f$ interferometer.

\subsection{Measurement of carrier envelope offset frequency}

We choose the SC spectrum corresponding to a pulse energy of $3.2 \mathrm{~nJ}$ for $f_{\text {ceo }}$ detection. One part of the SC beam is exported with a $50 \%$ broadband reflective mirror coating from 900 to $1,200 \mathrm{~nm}$ for other applications. The remaining is delivered into a typical $f$ - $2 f$ interferometer. The long-wavelength component around $1,156 \mathrm{~nm}$ is frequency-doubled and beats with the short-wavelength around $578 \mathrm{~nm}$ in the SC. A dispersion delay line consisting of two rectangular prisms is placed in the shortwavelength path. The $f_{\text {ceo }}$ of the $\mathrm{Yb}$-fiber laser system is determined by the heterodyne beat signal, which is detected by an avalanche photoelectric detector in the visible overlap region after a diffraction grating. Thanks to the good stability of the fiber ring cavity configuration, the $f_{\text {ceo }}$ center frequency is very stable with a resolution bandwidth of $100 \mathrm{kHz}$ over a long period of time. In this regard, it is much better than a Ti:sapphire-based comb whose $f_{\text {ceo }}$ usually drifts several megahertz in a minute. However, the linewidth is not as good. As shown in Fig. 5 , the free running $3 \mathrm{~dB}$ width of the $f_{\text {ceo }}$ is about $500 \mathrm{kHz}$ in our experiment, which is a little larger than that in previous results. The main reason for this comes from the dispersion characteristics of the Yb-fiber source. It is impossible to change the intra-cavity net dispersion in a commercial oscillator, which is a crucial factor in determining the $f_{\text {ceo }}$ noise. The narrowest linewidth always occurs in the case of near zero-dispersion [25]. In addition, the high power fiber amplifier may also worsen the quality of $f_{\text {ceo. }}$. Improvements in optimizing the dispersion and amplifier configuration should be helpful in reducing the noise and increasing the $f_{\text {ceo }}$ signal-to-noise ratio (SNR). 


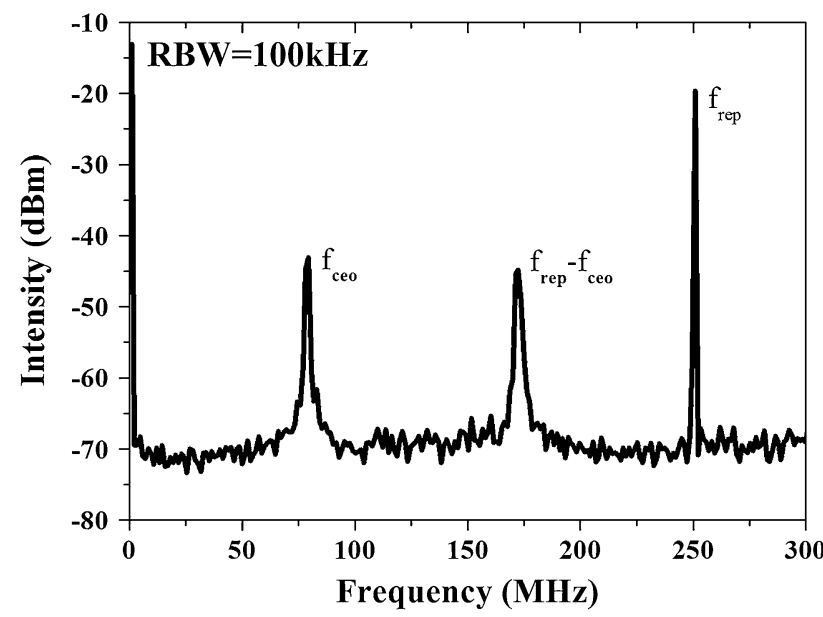

Fig. 5 The $f_{\text {ceo }}$ measurement with a typical $f$ - $2 f$ interferometer, $f_{\text {rep }}$ is the repetition rate

\section{Conclusion}

In conclusion, we have reported the generation of a broadband SC spanning more than one octave in a tapered single-mode fiber with a core diameter of $3 \mu \mathrm{m}$. The spectrum ranges from 550 to $1,400 \mathrm{~nm}$, and a SC power of over $1.3 \mathrm{~W}$ is obtained with a coupling efficiency of nearly $60 \%$. Based on such a broadband SC, the $f_{\text {ceo }}$ signal, which has an SNR of nearly $30 \mathrm{~dB}$ and linewidth of $500 \mathrm{kHz}$, has been characterized experimentally. Our results indicate that this tapered single-mode fiber could be applied in $f_{\text {ceo }}$ measurements and thus used to construct optical frequency combs. Inexpensive fabrication and the possibility to customize parameters are promising advantages for our optical frequency comb system, allowing more practical and stable operation in real application fields.

Acknowledgments We thank Dr. Wei Ding for helpful discussions. This work is partially supported by the National Basic Research Program of China (973 Program Grant No. 2012CB821304) and the National Natural Science Foundation of China (Grant Nos. 11078022 and 61378040).

Open Access This article is distributed under the terms of the Creative Commons Attribution License which permits any use, distribution, and reproduction in any medium, provided the original author(s) and the source are credited.

\section{References}

1. T. Morioka, K. Mori, M. Saruwatari, Elect. Lett. 29, 862 (1993)

2. I. Hartl, X.D. Li, C. Chudoba, R.K. Ghanta, T.H. Ko, J.G. Fujimoto, J.K. Ranka, R.S. Windeler, Opt. Lett. 26, 608 (2001)

3. S.T. Cundiff, J. Ye, Rev. Mod. Phys. 75, 325 (2003)

4. J.C. Knight, J. Broeng, T.A. Birks, P.S.J. Russel, Science 282, 1476 (1998)

5. J.K. Ranka, R.S. Windeler, A.J. Stentz, Opt. Lett. 25, 25 (2000)

6. J.M. Dudley, G. Genty, S. Coen, Rev. Mod. Phys. 78, 1135 (2006)

7. H.C. Nguyen, B.T. Kuhlmey, E.C. Mägi, M.J. Steel, P. Domachuk, C.L. Smith, B.J. Eggleton, Appl. Phys. B 81, 377 (2005)

8. S.P. Stark, T. Steinmetz, R.A. Probst, H. Hundertmark, T. Wilken, T.W. Hänsch, Th Udem, P.S.J. Russell, R. Holzwarth, Opt. Express 19, 15690 (2011)

9. T. Jiang, G. Wang, W. Zhang, C. Li, A. Wang, Z. Zhang, Opt. Lett. 38, 443 (2013)

10. T.A. Birks, Y.W. Li, J. Lightwave Technol. 10, 432 (1992)

11. T.A. Birks, W.J. Wadsworth, P.S.J. Russell, Opt. Lett. 25, 1415 (2000)

12. D.A. Akimov, A.A. Ivanov, M.V. Alfimov, S.N. Bagayev, T.A. Birks, W.J. Wadsworth, P.S.J. Russell, A.B. Fedotov, V.S. Pivtsov, A.A. Podshivalov, A.M. Zheltikov, Appl. Phys. B 74, 307 (2002)

13. J. Teipel, D. Türke, H. Giessen, A. Zintl, B. Braun, Opt. Express 13, 1734 (2005)

14. M. Rusu, S. Kivistö, C.B.E. Gawith, O.G. Okhotnikov, Opt. Express 13, 8547 (2005)

15. J. Kerttula, V. Filippov, V. Ustimchik, Y. Chamorovskii, O.G. Okhotnikov, Opt. Express 20, 25461 (2012)

16. Y.H. Li, Y.Y. Zhao, L.J. Wang, Opt. Lett. 37, 3441 (2012)

17. H.-Y. Lin, C.-H. Huang, G.-L. Cheng, N.-K. Chen, H.-C. Chui, Opt. Express 20, 21693 (2012)

18. Y. Yu, W. Ding, L. Gan, Z. Li, Q. Luo, S. Andrews, Opt. Express 22, 2528 (2014)

19. W.J. Wadsworth, A. Ortigosa-Blanch, J.C. Knight, T.A. Birks, T.-P.M. Man, P.S.J. Russell, J. Opt. Soc. Am. B 19, 2148 (2002)

20. L. Tong, J. Lou, E. Mazur, Opt. Express 12, 1025 (2004)

21. G.P. Agrawal, Nonlinear fiber optics, 3rd edn. (Academic Press, London, 1995)

22. A.V. Husakou, J. Herrmann, Phys. Rev. Lett. 87, 203901 (2001)

23. N. Akhmediev, M. Karlsson, Phys. Rev. A 51, 2602 (1995)

24. I. Cristiani, R. Tediosi, L. Tartara, V. Degiorgio, Opt. Express 12, 124 (2003)

25. L. Nugent-Glandorf, T.A. Johnson, Y. Kobayashi, S.A. Diddams, Opt. Lett. 36, 1578 (2011) 